\title{
Laparoscopic Sacrocolpopexy for Grade IV Pelvic Organs Prolapse with Associated Bilateral Pyelocaliceal Dilatation: The First Case Reported
}

\author{
Lessandro Curcio, Romolo Guida, Antonio C. Cunha, Juan Renteria, Ricardo Freire, Geraldo \\ Di Biase
}

Ipanema Federal Hospital (LC, ACC, JR, RF, GDB) and State Civil Servant Hospital (RG), Rio de Janeiro, Brazil

\begin{abstract}
Introduction: The main structures involved in maintaining the integrity of the pelvis include the ureterosacral ligament, pubocervical fascia, and the paracervical tissues. A compromise to any of these areas can promote a weakness leading to herniation or prolapse of the urethra, bladder, and/or rectum. Hydronephrosis can range from 5\% in first-degree to $40 \%$ in patients with third-degree prolapse. A variety of laparoscopic techniques has been described and some have used meshes as an integral part for the repair. This approach aims to restore normal voiding function while preserving female sexual function. Here, we provide a video of a pelvic organ prolapse (POP) female patient with bilateral pyelocaliceal dilation, that was corrected through a laparascopic sacrocolpopexy with mesh technique.

Methods: A 56 year-old female, complained of a ball in your vagina and just evacuated fezzes with aid of the fingers introduced inside the vagina. Her physic exam evidenced a grade 4 pelvic prolapse, bringing down rectum, bladder and urethra and probably kinking bilaterally the ureters, since IVP exam showed a dilated right kidney, almost without function, and the left with a delayed excretion. A laparoscopic correction of the POP was proposed. A 4 ports pneumoperitoneum was utilized. We dissected the retovaginal and bladder vaginal spaces. The mesh was sutured posterolaterally to the distal levator ani muscles, and centrally to central perineum tendon. Anteriorly, the mesh was sutured to the anterior vaginal wall and then passed through the broad ligaments. Both meshes were trimmed and sutured to the anterior longitudinal ligaments of the sacral promontory. The Douglas pouch and peritoneal incision were closed and a transobturator sub-urethral sling was positioned.

Results: The surgery lasted 240 minutes, with a minimum blood loss and just paracetamol was used for postoperative pain. She was discharged in 3 days and her 2 months contrast exam showed pelvic organs in a correct location and better contrast elimination of both kidneys. After a follow up of 12 months, she is continent, with no prolapse recurrence.

Conclusion: Despite some authors contesting the type of access required to correct the pelvic prolapse, undoubtedly the laparoscopic approach provides more anatomic detail, a clear surgical field, better cosmesis, and an early return to physical activity. Moreover, we showed that laparoscopic mesh approach for sacrocolpopexy is feasible, with a minimum morbidity, even in the context of bilateral hydronephrosis secondary to POP. To our knowledge, this is the first published case approached in such a manner in the scientific literature.
\end{abstract}

Int Braz,J Urol. 2010; 36 (Video \#6): 375_6

Available at: www.brazjurol.com.br/videos/may_june_2010/Curcio_375_376video.htm

\section{Correspondence address:}

Dr. Lessandro Curcio

Ipanema Federal Hospital

Av. Ayton Senna, 1850 / 223

22790-700, Rio de Janeiro, RJ, Brazil

E-mail: lessandrocg@ig.com.br 


\section{EDITORIAL COMMENT}

Curcio et al. have described and shown nice detail pertaining to the Laparoscopic Sacrocolpopexy for Grade IV pelvic organ prolapse. The authors also delineate the relationship between the presence of preoperative bilateral pyelocaliceal dilation and the subsequent resolution postoperatively. This observation is somewhat novel.
A nice review of the pertinent anatomy and technique is explained both in the written abstract as well as in the video. Finally, technical points of the actual repair are clear, concise and the satisfactory postoperative information is reported.

Dr. Rafael E. Carrion Associate Professor of Urology Department of Urology USF Health College of Medicine

Tampa, Florida, USA E-mail: rcarrion@health.usf.edu 